\title{
Therapeutic plasma exchange in treating multi-organ injury at the later stage of mushroom poisoning: Three case reports
}

\author{
Li Chen ${ }^{1}$, Benxiu Teng ${ }^{1}$, Juan Luo ${ }^{1}$, Xianlong Ling $^{2}$, Haiyan Zhao ${ }^{2}$, Qing Zhang ${ }^{3}$, Yuzhu Zeng ${ }^{1}$, \\ Gaojie Yuan ${ }^{1}$, Fang Tan ${ }^{1}$, Yin Ye ${ }^{1}$, Wenhan Duan ${ }^{1}$, Zhongjun $\mathrm{Li}^{1}$. \\ ${ }^{1}$ Department of Blood Transfusion, ${ }^{2}$ Department of Gastroenterology, \\ ${ }^{3}$ Department of Respiration, The Second Affiliated Hospital, Third Military Medical University, Chongqing, 400037 China
}

\begin{abstract}
Ingestion of Amanita phalloides, a deadly wild mushroom, can cause multi-organic injury. The value of therapeutic plasma exchange (TPE) in treating mushroom poisoning was controversial, especially for those patients at the later stage. We reported three cases of acute liver injury, coagulopathy, and hepatic encephalopathy secondary to mushroom poisoning at the later stage. Therapeutic plasma exchange (TPE) session was repeated $2 \sim 5$ times depending on the patients' conditions. Pathological plasma ranging from 1600 to $1800 \mathrm{~mL}$ was exchanged for each session with fresh frozen plasma and normal saline based on the patients' body weight. Patients' liver function, coagulation function, and clinical symptoms were immediately improved upon TPE treatment. TPE is a reliable therapy for patients with acute liver injury, coagulopathy, and hepatic encephalopathy secondary to mushroom poisoning at the later stage of poisoning.
\end{abstract}

Keywords: Mushroom poisoning, Therapeutic plasma exchange, Acute liver injury, Coagulopathy, Hepatic encephalopathy

\section{INTRODUCTION}

It is well known that poisoning caused by mushroom Amanita phalloides can result in acute liver injury. Furthermore, acute hepatic failure, coagulopathy, hemolytic anemia, and even hepatic encephalopathy with high mortality can happen if treatment is not conducted in a timely fashion ${ }^{[1,2]}$. Some cases and retrospective studies have shown that adjunctive TPE therapy combined with conservative treatment results in lower mortality ${ }^{[3,4]}$. However, there are still debates over the value of TPE in treating mushroom poisoning because no randomized and controlled trial has been

\footnotetext{
*Correspondence to: Zhongjun Li, PhD (johnneyusc@gmail.com) at Department of Blood Transfusion, The Second Affiliated Hospital, Third Military Medical University, Chongqing, China. Phone: 86023-68755319. Fax: 086-023-68755319.
}

conducted to compare TPE with a standard therapy ${ }^{[5]}$. Thus, TPE is a very weak recommendation (Grade 2C) by the American Society for Apheresis (ASFA) for treating mushroom poisoning ${ }^{[6]}$. It is recommended to initiate TPE during the first 24-48 hours after being poisoned ${ }^{[5]}$. The mortality of patients receiving TPE therapy 72 hours after being poisoned was 14.67 times higher than that of patients receiving TPE within 72 hours after being poisoned ${ }^{[7]}$.

We reported here three cases of acute liver injury, coagulopathy, and hepatic encephalopathy secondary to mushroom poisoning. Three patients were successfully treated using TPE 96-144 hours after the poisoning. Their liver function, coagulation function, and clinical symptoms were immediately improved after the TPE treatment. Our reports suggested that TPE is an indispensable treatment for patients at the later stage after being poisoned by Amanita phal- 
loides.

\section{Methods}

The patients signed informed consent for the publication of this case report. And this study was approved by the ethics committee of Xinqiao Hospital.

\section{CASE REPORTS}

\section{Case 1}

A 79-year-old woman (body weight: $40 \mathrm{~kg}$ ) was transferred to our hospital on 17 June 2015 because of persistent vomiting and dizziness for 4 days after the ingestion of some wild mushroom. Laboratory examination showed that she had liver dysfunction and coagulation disorder. Liver protective treatment has been conducted at a local hospital. However, no improvement was observed. Laboratory results after being admitted to our hospital revealed as follows: ALT $4051 \mathrm{IU} / \mathrm{L}$, AST $4312 \mathrm{IU} / \mathrm{L}$, TBIL $30.5 \mu \mathrm{mol} / \mathrm{L}$, DBIL $17.1 \mu \mathrm{mol} / \mathrm{L}$, PT $25.0 \mathrm{~s}$, PTA 26.1\%, INR 2.14, and APTT $48.6 \mathrm{~s}$. Fluid replenishment and supporting therapy were conducted immediately. Dexamethasone acetate injection was used to relieve the pathologic immune cascade reaction, and TPE was performed. One thousand and six hundred milliliter pathological plasma was exchanged with $1000 \mathrm{~mL}$ fresh frozen plasma and $600 \mathrm{~mL}$ normal saline once a day for two days. The blood flow rate was $50 \mathrm{~mL} / \mathrm{min}$. Laboratory results following the TPE on day lwere ALT 578 IU/L, AST 128 IU/L, TBIL $47.8 \mu \mathrm{mol} / \mathrm{L}$, DBIL 20.9 $\mu \mathrm{mol} / \mathrm{L}$, PT $13.4 \mathrm{~s}$, PTA $64.1 \%$, INR 1.15, and APTT 39.8 s (Table 1). Apparently, the liver function and coagulation function improved remarkably following the TPE. The vomiting and dizziness were also relieved gradually. On day 5 , the patient complained of an epigastric pain. Gastroscopy showed one $0.5 \mathrm{~cm}$ diameter ulcer at the duodenal ampulla. Anti-peptic ulcer therapy was further conducted and the patient left the hospital on day 10 without any sequelae of mushroom poisoning.

\section{Case 2}

A 48-year-old man (body weight: $45 \mathrm{~kg}$ ), the son of the patient in case 1, was transferred to our hospital with persistent vomiting, dizziness and palpitation for 4 days. He ate more mushrooms than his mother and presented more severe symptoms. Laboratory results showed the following values: ALT $4561 \mathrm{IU} / \mathrm{L}$, AST $5891 \mathrm{IU} / \mathrm{L}$, TBIL $54.2 \mu \mathrm{mol} / \mathrm{L}$, DBIL $27.1 \mu \mathrm{mol} / \mathrm{L}$, PT $42.5 \mathrm{~s}$, PTA $13.5 \%$, INR 3.62, and APTT $69.2 \mathrm{~s}$. In addition to fluid replenishment, adrenocortical hormones treatment, and supporting therapy, TPE was also performed us- ing $1200 \mathrm{~mL}$ fresh frozen plasma and $600 \mathrm{~mL}$ normal saline to replace $1800 \mathrm{~mL}$ pathological plasma, with a blood flow rate at $50 \mathrm{~mL} / \mathrm{min}$. After two sessions of TPE, the laboratory blood results were ALT $1078 \mathrm{IU} / \mathrm{L}$, AST $187 \mathrm{IU} / \mathrm{L}$, TBIL $119 \mu \mathrm{mol} / \mathrm{L}$, DBIL $51.8 \mu \mathrm{mol} / \mathrm{L}$, PT 14.4 s, PTA 58.2 \%, INR 1.24, and APTT $31.2 \mathrm{~s}$ (Table 1). The liver function and coagulation function were also remarkably improved, and no vomiting was observed any more. However, the TBIL increased unexpectedly. Ademetionine 1, 4-Butane disulfonate, and $\mathrm{L}-$ ornithine- $\mathrm{L}-$ aspartate were used for antiTBIL treatment. Two days later, the TBIL returned to $44.6 \mu \mathrm{mol} / \mathrm{L}$ and all related symptoms were obviously relieved. The patient left the hospital with his mother on the tenth day of hospitalization.

\section{Case 3}

A $64-$ year-old man (body weight: $60 \mathrm{~kg}$ ) was transferred to our hospital. He had eaten several wild mushrooms 6 days before, and then presented with diarrhea, nausea, vomiting, and disturbance of consciousness. The symptoms were slightly relieved after normal transfusion therapy for two days at a local hospital. The diarrhea, nausea and vomiting disappeared. However, his condition got worse on $3 \mathrm{Sep}-$ tember 2015. He presented dysphoria, jaundice, and could not answer any questions when he was admitted to our hospital. Laboratory examination showed as follows: ALT $3978 \mathrm{IU} / \mathrm{L}$, AST $1229 \mathrm{IU} / \mathrm{L}$, TBIL $211.1 \mu \mathrm{mol} / \mathrm{L}$, DBIL $95.8 \mu \mathrm{mol} / \mathrm{L}, \mathrm{NH}_{3} 132 \mu \mathrm{mol} / \mathrm{L}$, PT 43.5 s, PTA $14.4 \%$, INR 3.11, and APTT $74.2 \mathrm{~s}$. Propofol injection was used for appeasement. Compound tinidazole solution, lactulose oral solution, and compound amino acid $3 \mathrm{AA}$ injection were used to decrease blood $\mathrm{NH}_{3}$. One thousand and eight hundred milliliter poisoned plasma was exchanged with $800 \mathrm{~mL}$ ordinary frozen plasma, $400 \mathrm{~mL}$ fresh frozen plasma, and $600 \mathrm{~mL}$ normal saline once a day for five days. After each TPE session, 10 units of cryoprecipitate were further used to improve the coagulation function. Five days following the TPE the laboratory blood results were ALT 135 IU/L, AST 42 IU/ L, TBIL $159.3 \mu \mathrm{mol} / \mathrm{L}$, DBIL $85.8 \mu \mathrm{mol} / \mathrm{L}, \mathrm{NH}_{3} 78$ $\mu \mathrm{mol} / \mathrm{L}$, PT $16.1 \mathrm{~s}$, PTA $48.6 \%$, INR 1.38, and APTT 56.9 s (Table 1). His liver function and coagulation function improved remarkably. The patient's symptoms were largely ameliorated. However, the levels of TBIL and blood $\mathrm{NH}_{3}$ were still high. Abdomen erect position $\mathrm{X}$-ray examination showed intestinal meteorism without obstruction. Blood electrolyte test indicated hypokalemia. Blind enema and $10 \% \mathrm{~K}$-Lor were utilized to improve the excretory function. The level of blood $\mathrm{NH}_{3}$ returned to normal by the tenth day 
after admission. However, the level of TBIL increased to $206 \mu \mathrm{mol} / \mathrm{L}$ again. Progressively decrease of haemoglobin and the positive result of direct antiglobulin test indicated that the patient had hemolytic anemia. Pathologic haemolysis resulted in the re-elevation of TBIL. Thus, Prednisone was then used for anti-TBIL treatment. The level of TBIL returned to $110 \mu \mathrm{mol} / \mathrm{L}$ on the 19th day and the jaundice alleviated gradually. Three days later, the level of TBIL returned to $55.8 \mu \mathrm{mol} / \mathrm{L}$, and no further related symptoms were observed and the patient left the hospital without any sequelae.

\section{DISCUSSION}

The target with TPE is typically a single constituent of plasma mainly including albumin or globulin ${ }^{[8]}$. However, amanitins, the active compounds in mushroom poison, does not bind to albumin ${ }^{[9]}$. The efficiency of toxin removal carried out by TPE was lower than forced diuresis ${ }^{[4]}$. Thus, It was supposed that any benefit of TPE might derive from active removal of harmful endogenous metabolites that result from amatoxin induced cellular necrosis ${ }^{[10]}$. As showing in the Table 1, TPE treatment can efficiently decrease the level of transaminases in the blood and improve the coagulation function. Thus, TPE treatment is quite necessary for patients with acute liver injury (case 1 and 2) and hepatic encephalopathy (case 3) secondary to mushroom poisoning, especially for those who missed the initial 72 hours of treatment as they normally had highly elevated transaminases level and impaired coagulation function. However, TPE had little effect on the reduction of bilirubin (Table 1). Further anti-TBIL treatment was critical when TPE was conducted.

By using centrifugal plasma separator, we could extract and exchange $75 \%$ 100\% of the pathological plasma each time which guaranteed the high efficiency of the removal of those harmful endogenous metabolites. However, the recovery of blood coagulation factors and the metabolism of bilirubin were still depended on liver regeneration. To correct the coagulopathy, fresh frozen plasma was used as the replacement fluid during TPE. Cryoprecipitation was further utilized if the fibrinogen decreased progressively. TPE combined with anti-TBIL treatment not only temporarily replaced the synthesis and detoxification function of the impaired liver, but also supported liver regeneration for long-term recovery ${ }^{[11]}$. Most recently, a randomized, controlled multicenter trial demonstrated that treatment with high-volume TPE ameliorate multi-organ dysfunction and improves outcome in patients with acute liver failure ${ }^{[12,13]}$, which indirectly supported the use of TPE in the later stage of mushroom poisoning when multi organs were impaired. However, the component and volume of the replacement fluid and the frequency of TPE should be further optimized.

\section{Declaration of interests}

We declare no competing interests.

\section{Acknowledgments}

This research was supported by the Youth Scientist Foundation of Chongqing (CSTC 2013JCYJJQ10001).

Table 1 Laboratory results of patients pre- and post-TPE treatment

\begin{tabular}{|c|c|c|c|c|c|c|c|c|c|c|}
\hline \multicolumn{2}{|c|}{ Treatment } & ALT(IU/L) & AST(IU/L) & $\begin{array}{c}\text { TBIL } \\
(\mu \mathrm{mol} / \mathrm{L})\end{array}$ & $\begin{array}{c}\text { DBIL } \\
(\mu \mathrm{mol} / \mathrm{L})\end{array}$ & $\begin{array}{c}\mathrm{NH}_{3} \\
(\mu \mathrm{mol} / \mathrm{L})\end{array}$ & $\mathrm{PT}(\mathrm{s})$ & $\operatorname{PTA}(\%)$ & INR & APTT(s) \\
\hline \multicolumn{2}{|c|}{ Normal range } & $0-40$ & $0-40$ & $1.7-17.1$ & $0-7$ & $2-60$ & $9-14$ & $70-120$ & $0.7-1.3$ & $20-40$ \\
\hline \multirow[t]{2}{*}{ Case 1} & Before & 4051 & 4312 & 30.5 & 17.1 & - & 25.0 & 26.1 & 2.14 & 48.6 \\
\hline & After & 578 & 128 & 47.8 & 20.9 & - & 13.4 & 64.1 & 1.15 & 39.8 \\
\hline \multirow[t]{2}{*}{ Case 2} & Before & 4561 & 5891 & 54.2 & 27.1 & - & 42.5 & 13.5 & 3.62 & 69.2 \\
\hline & After & 1078 & 187 & 119 & 51.8 & - & 14.4 & 58.2 & 1.24 & 31.2 \\
\hline Case 3 & After & 135 & 42 & 159.3 & 85.8 & 78 & 16.1 & 48.6 & 1.38 & 56.9 \\
\hline
\end{tabular}

ALT, aspartate aminotransferase; AST, aspartate aminotransferase; TBIL, total bilirubin; DBIL, direct bilirubin; PT, prothrombin time; PTA, prothrombin activity; INR, international normalized ratio; APTT, activated partial thromboplastin time.

\section{References}

[1] Tolentino P. Liver failure. Paediatrician, 1978;7:166-75.

[2] Kuruvilla J, Gupta V, Gill KS, Lipton JH. Association of familial leukemia with HLA Cw3: is it real? Leukemia \& lymphoma, 2003;44:309-11.

[3] Jankowska I, Grenda R, Ryzko J, et al. [Evaluation of plasmapheresis utility in treatment of poisoning with
Amanita phalloides]. Polski tygodnik lekarski (Warsaw, Poland:1960), 1993;48:427-9.

[4] Jander S, Bischoff J. Treatment of Amanita phalloides poisoning: I. Retrospective evaluation of plasmapheresis in 21 patients. Therapeutic apheresis : official journal of the International Society for Apheresis and the Japanese 
Society for Apheresis, 2000;4:303-7.

[5] Schutt RC, Ronco C, Rosner MH. The role of therapeutic plasma exchange in poisonings and intoxications. Seminars in dialysis, 2012;25:201-6.

[6] Schwartz J, Winters JL, Padmanabhan A, et al. Guidelines on the Use of Therapeutic Apheresis in Clinical Practice-Evidence-Based Approach from the Writing Committee of the American Society for Apheresis: The Sixth Special Issue. J Clin Apheresis, 2013;28:145-284.

[7] Gan W-m, Zhang X-g. Curative effect and timing of plasma exchange in the treatment of patients with mushroom poisoning of severe liver damage. China Journal of Emergency Resuscitation and Disaster Medicine, 2012;7:453-60.

[8] Oguz FS, Kalayoglu S, Diler AS, et al. HLA system affects the age-at-onset in chronic myeloid leukemia. American journal of hematology, 2003;73:256-62.

[9] Fiume L, Sperti S, Montanaro L, et al. Amanitins do not bind to serum albumin. Lancet, 1977;1:1111.
[10] Jander S, Bischoff J, Woodcock BG. Plasmapheresis in the treatment of Amanita phalloides poisoning: II . A review and recommendations. Therapeutic apheresis : official journal of the International Society for Apheresis and the Japanese Society for Apheresis, 2000;4:308-12.

[11] Ozcay F, Baskin E, Ozdemir N, et al. Fulminant liver failure secondary to mushroom poisoning in children: importance of early referral to a liver transplantation unit. Pediatric transplantation, 2006;10:259-65.

[12] Larsen FS, Schmidt LE, Bernsmeier C, et al. Highvolume plasma exchange in patients with acute liver failure: An open randomised controlled trial. Journal of hepatology, 2016;64(1):69-78.

[13] Karvellas CJ, Todd Stravitz R. High Volume Plasma Exchange in Acute Liver Failure: Dampening the inflammatory cascade? Journal of hepatology, 2016;64(1):10-2.

(Received 04 February 2017, Revised 22 February 2017, Accepted 06 March 2017) 